\title{
Clinical Characteristics and Natural History of Hypertrophic Cardiomyopathy With Midventricular Obstruction
}

\author{
Georgios K. Efthimiadis, MD, PhD; Efstathios D. Pagourelias, MD, PhD; \\ Despoina Parcharidou, MD, PhD; Thomas Gossios, MD; Vasileios Kamperidis, MD; \\ Efstratios K. Theofilogiannakos, MD; Zoi Pappa, MD; Soultana Meditskou, MD, PhD; \\ Stavros Hadjimiltiades, MD, PhD; Christodoulos Pliakos, MD, PhD; \\ Haralampos Karvounis, MD, PhD; Ioannis H. Styliadis, MD, PhD
}

\begin{abstract}
Background: The prevalence, clinical characteristics and natural history of patients with hypertrophic cardiomyopathy (HCM) and midventricular obstruction (MVO) have not been adequately studied.
\end{abstract}

\begin{abstract}
Methods and Results: A single-center cohort consisting of 423 patients (mean age, $49.3 \pm 17.2$ years; $66.2 \%$ male) was thoroughly followed up for a median of 84 months (7 years; range, $6-480$ months). MVO, characterized by the echocardiographic appearance of midventricular muscular apposition with a simultaneous mid-cavitary gradient $\geq 30 \mathrm{mmHg}$, was identified in 34 patients (8\%). Patients with MVO tended to be more symptomatic during their initial evaluation ( $>90 \%$ presented with NYHA class $\geq \mathrm{Il}$ ) compared to the rest of the HCM cohort. Apical aneurysm formation was identified in more than one-fourth of patients with MVO (26.5\%), being a characteristic of the group. On multivariate Cox regression hazard analysis, presence of MVO strongly predicted progression to end-stage (burnt out) $\mathrm{HCM}$ and related heart failure (HF) deaths (hazard ratio, [HR], 2.62; 95\% confidence interval [Cl]: 1.2-8.8; $\mathrm{P}=0.047$ ), as well as sudden death and associated lethal arrhythmic events (HR, 3.3; 95\% Cl: 1.26-8.85; P=0.016).
\end{abstract}

Conclusions: MVO is a distinct phenotype of HCM associated with unfavorable prognosis in terms of end-stage $\mathrm{HCM}$, sudden death and lethal arrhythmic events. The high adverse outcome rate necessitates early recognition of MVO and appropriate therapeutic interventions. (Circ $J$ 2013; 77: 2366-2374)

Key Words: Heart failure; Hypertrophic cardiomyopathy; Midventricular obstruction; Sudden death

$\mathbf{H}$ ypertrophic cardiomyopathy ( $\mathrm{HCM})$ is an inherited cardiovascular disorder of great genetic heterogeneity $^{1,2}$ and has a prevalence of $0.2 \%$ in the general population. ${ }^{3}$ Its main morphological characteristics include fibrosis and disarray at the myocyte level along with segmental hypertrophy and/or obstruction at different intracavitary points (either subaortic or midventricular). ${ }^{3}$ Impedance to flow at the middle of the left ventricle (LV), so-called midventricular obstruction (MVO), is a distinct and recognized phenotype of HCM, occurring as a result of segmental mid-septal hypertrophy and hypercontractility of the lateral ventricular wall along with the misplacement of the hypertrophied papillary muscles. ${ }^{4}$ Although obstruction at the subaortic level has been adequately studied to date, the clinical features and natural history of patients with MVO are undetermined, because previous de- scriptions have been confined to case reports or small patient series, ${ }^{4-11}$ while only a recent study has described in a more systematic manner the course of MVO in an Asian population. ${ }^{12}$ Given that HCM patients with MVO remain underevaluated, we studied the prevalence, clinical profile and natural history of a subgroup of patients with MVO, selected from a single-center cohort of patients with HCM.

\section{Patient Selection}

From February 1992 to June 2011, 423 consecutive patients with documented HCM were assessed at Cardiomyopathies Center AHEPA University Hospital, Thessaloniki, Greece and were followed up at AHEPA University Hospital. Follow-up

Received December 20, 2012; revised manuscript received March 30, 2013; accepted April 23, 2013; released online May 31, 2013 Time for primary review: 27 days

Cardiomyopathies Center, First Cardiology Department (G.K.E., E.D.P., D.P., T.G., V.K., E.K.T., Z.P., S.H., H.K., I.H.S.), First Department of Internal Medicine (C.P.), AHEPA University Hospital, Thessaloniki; and Laboratory of Histology and Embryology, Medical School, Aristotle University of Thessaloniki, Thessaloniki (S.M.), Greece

Mailing address: Efstathios D. Pagourelias, MD, PhD, Cardiomyopathies Center, First Cardiology Department, AHEPA University Hospital, Aristotle University of Thessaloniki, Stil. Kyriakidi 1 Str, PC 54636, Thessaloniki, Greece. E-mail: statpag@yahoo.gr

ISSN-1346-9843 doi:10.1253/circj.CJ-12-1561

All rights are reserved to the Japanese Circulation Society. For permissions, please e-mail: cj@j-circ.or.jp 


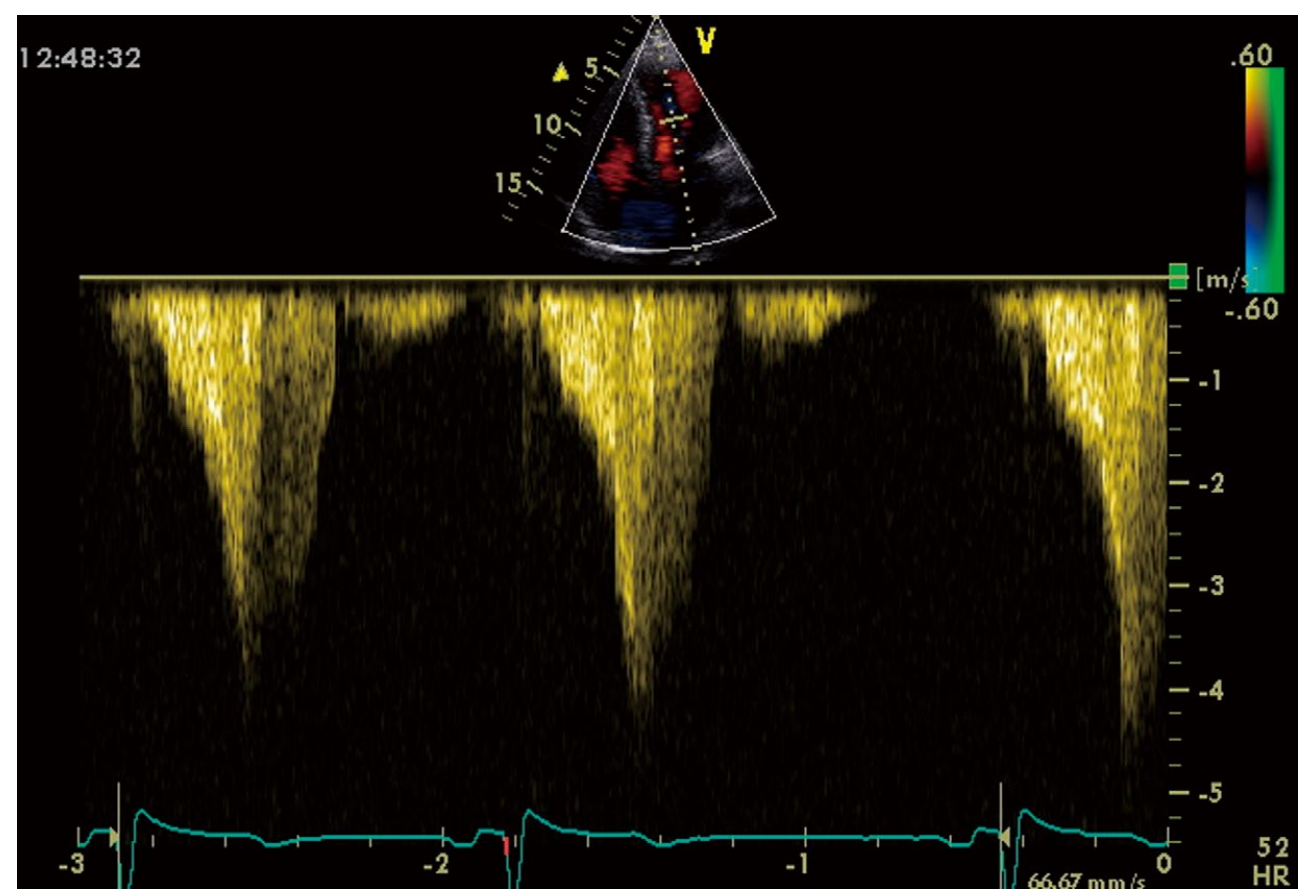

Figure 1. Continuous wave Doppler recording with the vector placed in the middle of the left ventricle. A dagger-shaped waveform is obtained, showing a high midventricular gradient with an estimated peak velocity of $4.5 \mathrm{~m} / \mathrm{s}$.

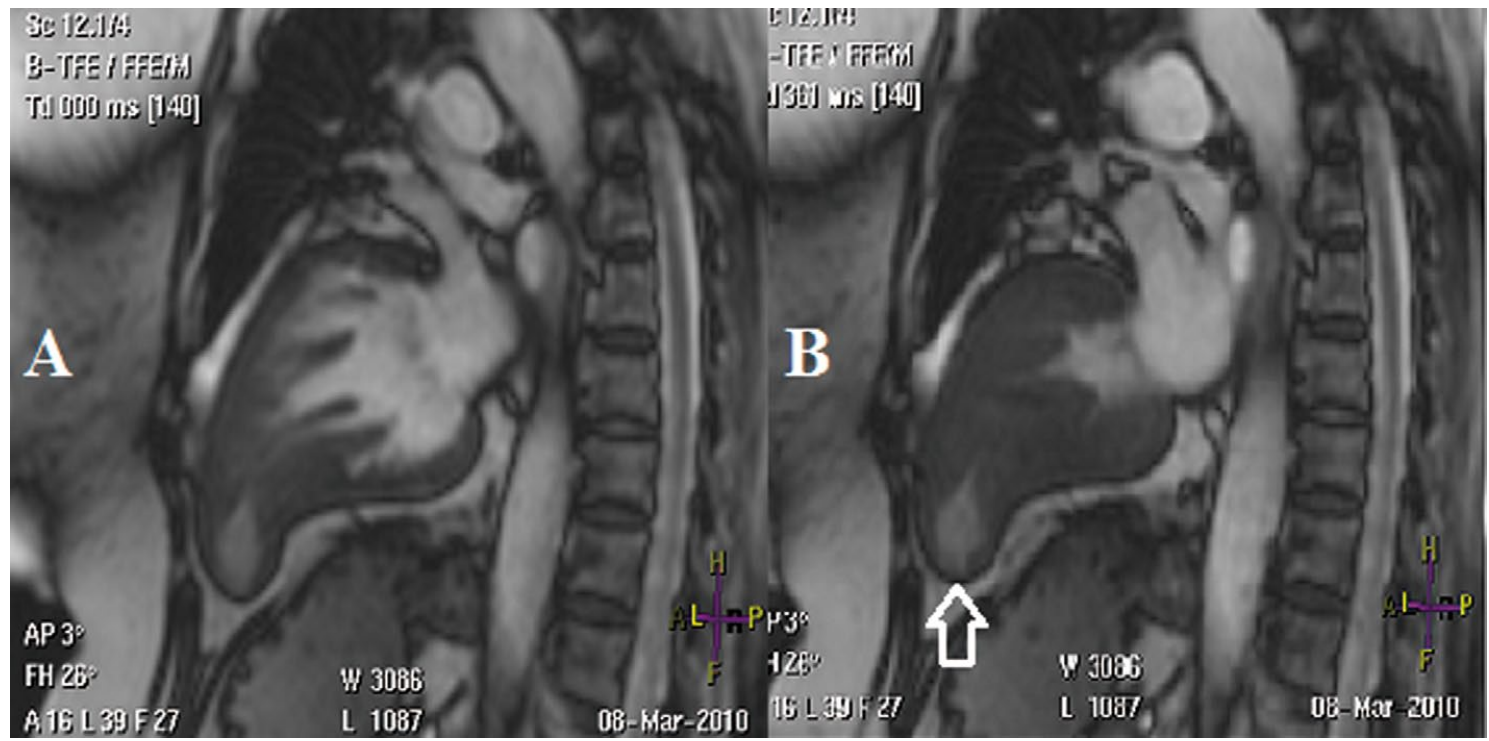

Figure 2. (A) Two-chamber magnetic resonance imaging view of left ventricle (LV) during diastole with midventricular hypertrophy leading to hourglass shape of the ventricle. (B) LV during systole. Absolute mid-cavity obliteration is evident along with the formation of an apical aneurysm (arrow).

extended from the point at which the initial diagnosis was made, to the most recent evaluation in surviving patients (either in outpatient clinics or by telephone review), or to death/ endpoint. The study protocol complied with the Declaration of Helsinki. The institutional ethics committee approved the re- search protocol, and the study participants provided written informed consent at their first evaluation.

\section{Definitions}

HCM was defined as the echocardiographic appearance of LV 
maximum wall thickness $\geq 15 \mathrm{~mm}$, in the absence of any other cause capable of producing such hypertrophy; ${ }^{3}$ or the presence of maximum wall thickness 13 or $14 \mathrm{~mm}$ and a positive family history for HCM and/or electrocardiographic changes compatible with HCM. . $^{3,13}$

Left ventricular outflow tract obstruction (LVOTO), caused by anterior motion of mitral valve leaflets, was defined as present when the estimated peak instantaneous gradient on continuous Doppler application was $\geq 30 \mathrm{mmHg}$. ${ }^{3}$

MVO was defined as midventricular gradient $\geq 30 \mathrm{mmHg}$ (Figure 1), with a simultaneous appearance of midventricular muscular apposition, creating an hourglass shape of the LV, on echocardiography or magnetic resonance imaging (MRI; Figure 2). ${ }^{12}$ All patients included in this study were thoroughly assessed, both during initial evaluation and follow-up, so as to detect midventricular gradients. Patients with both LVOTO and MVO were included in the MVO group, because their phenotypic characteristics and course were closer to those of the MVO group. LV apical aneurysm was defined as a discrete, thin-walled dyskinetic or akinetic apex with a relatively wide communication to the LV cavity (Figure 2). ${ }^{12}$

\section{Clinical Outcomes and Study Endpoints}

On the basis of previously published data 5 non-interventional clinical features were defined as risk factors for sudden death: (1) syncope; (2) premature sudden death; (3) recurrent episodes of non-sustained ventricular tachycardia; (4) abnormal blood pressure response during exercise; and (5) severe hypertrophy $\geq 30 \mathrm{~mm}^{3}$

Four modes of HCM-related events were defined as clinical endpoints: (1) all-cause mortality including deaths due to any cause during follow-up; (2) cardiovascular mortality accounting for deaths due to heart failure (HF), stroke or sudden death; (3) the combined cardiovascular endpoint fulfilled by patients who experienced end-stage or burnt out HCM (meaning an ejection fraction $<50 \%$ and dilated phenotype) or died due to HF; and (4) the surrogate sudden death endpoint, which consisted of sudden death events along with potentially lethal arrhythmic incidents such as ventricular tachycardia or fibrillation, resuscitated cardiac arrest and appropriate implantable defibrillator (ICD) discharge.

\section{Baseline Evaluation}

Baseline patient evaluation included personal and family history, clinical evaluation, 12-lead electrocardiogram, transthoracic echocardiography, 24-h electrocardiographic monitoring, and upright treadmill exercise stress test. Echocardiography was performed using commercially available ultrasound equipment and included M-mode, 2-D, pulsed- and continuous-wave Doppler echocardiography, and tissue Doppler imaging. ${ }^{14,15}$ Segmental LV hypertrophy was measured on 2-D echocardiography parasternal short-axis views, according to previously described methods. Basal subaortic and midventricular gradients were quantified using continuous-wave Doppler echocardiography under resting conditions (Figure 1) with auxiliary use of color Doppler imaging to localize the turbulent flow and point of obstruction and were thoroughly searched for in all patients. ${ }^{14}$ Specifically, concerning MVO, mid-cavitary gradient was prospectively evaluated in all of the present patients, from enrolment, with the continuous wave vector placed in the middle of the LV. Simultaneously, any signs of mid-cavitary turbulent flow were carefully searched for.

The patients also underwent a symptom-limited upright exercise test using the Bruce protocol. Finally, 24-h Holter electrocardiographic recordings were made while the patients performed their ordinary daily activities.

\section{Cardiac MRI}

Cardiac MRI was performed in 100 patients in the present cohort (15/34 MVO patients, 44.1\%; and 85/389 HCM \pm LVOTO patients, 21.9\%). Cardiac MRI was carried out with a 1.5-T scanner (Philips INTERA CV). Electrocardiographic gated breath-hold steady-state free precession cine images were obtained in 2-, 3- and 4-chamber views as well as in 8-mm shortaxis slices (no gap) from the base to the apex. Gadolinium was given in a $0.2-\mathrm{ml} / \mathrm{kg}$ bolus dose. Ten minutes later, gradient echo sequences were acquired, with inversion recovery in multiple slices to assess the presence of myocardial fibrosis. The presence or absence of fibrosis was assessed on visual assessment of delayed gadolinium enhancement (without thresholding), because visual assessment has been shown to correlate well with the use of a threshold of signal intensity of $6 \mathrm{SD}$ above the mean for normal myocardium. ${ }^{17}$

\section{Statistical Analysis}

Analysis was performed using SPSS Statistics, version 19.0 (IBM, SPSS Statistics, Chicago, IL, USA). Data are presented as mean $\pm \mathrm{SD}$ for continuous variables and as counts and or percentages for categorical ones. Normal distribution of data was tested using Kolmogorov-Smirnov test. Comparison of variables was performed with Student's t-test or 1-way analysis of variance (ANOVA) with post-hoc Bonferroni analysis for groups more than 2. Chi squared or Fisher exact test (when an expected value was $<5$ ) were used to compare nominally scaled variables.

Cumulative event rates through follow-up were estimated using the Kaplan-Meier method (5-year survival with $95 \%$ confidence intervals ([CIs] are provided for each group concerning each endpoint) and differences in survival or endpoint rates were assessed on log-rank test. To estimate hazard ratios (HRs) for reaching the defined endpoints and identify independent predictors of the study outcomes, univariate and multivariate Cox proportional hazard models were adopted. Clinical variables for univariate analysis were chosen according to previously published data. Variables demonstrating statistical significance on univariate analysis were then used for in multivariate Cox proportional hazard models. The influences of profile, interaction and co-linearity in the multivariate regression models were examined using regression diagnostic analysis. Two-tailed $\mathrm{P}<0.05$ was considered statistically significant.

\section{Results}

\section{Baseline Demographic and Clinical Characteristics}

MVO was identified in 34 of the 423 present HCM patients (8\%). Among the 34 patients with MVO there were 14 patients (41.2\%) who had obstruction both at the midventricular level and at the outflow tract of the LV. Baseline characteristics and comparison between groups (HCM, n=301; HCM with LVOTO, $\mathrm{n}=88$; and HCM with MVO, $\mathrm{n}=34$ ) are given in Table 1. Compared to HCM patients without obstruction and HCM patients with LVOTO, patients with MVO tended to be mostly female $(62.9 \%)$ and more symptomatic during initial evaluation $(97.1 \%)$. Dyspnea was by far the dominating symptom at presentation $(15 / 34,44 \%)$. Approximately $90 \%$ of the HCM patients with MVO presented with New York Heart Association (NYHA) class $\geq \mathrm{II}$; at the same time, 1 of $2 \mathrm{HCM}$ patients without obstruction were in NYHA class I. The formation of apical aneurysms seemed to be a characteristic of patients with MVO because $26.5 \%$ of them had aneurysms (compared to 


\begin{tabular}{|c|c|c|c|c|}
\hline & $\operatorname{HCM}(n=301)$ & LVOTO $(n=88)$ & MVO (n=34) & P-value \\
\hline Gender (male) & $210(69.8)$ & $54(61.4)$ & $16(47.1)$ & 0.017 \\
\hline Age at diagnosis (years) & $47.7 \pm 16.9$ & $52.5 \pm 17.1$ & $50.1 \pm 16.6$ & 0.068 \\
\hline Age at first evaluation (years) & $52.1 \pm 15.6$ & $57.4 \pm 15.2$ & $54.9 \pm 16.6$ & $0.017^{*}$ \\
\hline Symptoms at presentation & $202(67.1)$ & $75(85.2)$ & $33(97.1)$ & $<0.0005$ \\
\hline Normal ECG & $34(11.3)$ & $1(1.1)$ & $1(2.9)$ & 0.005 \\
\hline $\mathrm{FH} \mathrm{HCM}$ & $101(33.6)$ & $26(29.5)$ & $11(32.4)$ & 0.779 \\
\hline \multicolumn{5}{|l|}{ SCD risk factors } \\
\hline MWT >3.0 (cm) & $10(3.3)$ & $16(18.2)$ & $4(11.8)$ & $<0.0005$ \\
\hline NSVT & $27(9)$ & $9(10.2)$ & $7(20.6)$ & 0.105 \\
\hline ABPR & 37 (12.3) & $14(15.9)$ & $6(17.6)$ & 0.518 \\
\hline Syncope & $34(11.3)$ & $15(17)$ & $9(26.5)$ & 0.03 \\
\hline FH SCD & $31(10.9)$ & $8(9.1)$ & $7(20.6)$ & 0.157 \\
\hline \multicolumn{5}{|l|}{ NYHA class diagnosis } \\
\hline I & $141(46.8)$ & $14(13.9)$ & $3(8.8)$ & $<0.0005$ \\
\hline II & $129(42.9)$ & $46(52.3)$ & $20(58.8)$ & \\
\hline III & $27(9)$ & $26(29.5)$ & $10(29.4)$ & \\
\hline IV & $4(1.3)$ & $2(2.3)$ & $1(2.9)$ & \\
\hline \multicolumn{5}{|l|}{$\mathrm{AF}$} \\
\hline Paroxysmal & 45 (18.3) & $8(9.1)$ & $12(35.3)$ & 0.001 \\
\hline Permanent & $25(8.3)$ & $3(3.4)$ & $2(5.9)$ & 0.278 \\
\hline Progression to end-stage $\mathrm{HCM}$ & $21(7)$ & $4(4.5)$ & $4(11.8)$ & 0.363 \\
\hline Apical aneurysm & $1(0.3)$ & $0(0)$ & $9(26.5)$ & $<0.0005$ \\
\hline Stroke & $27(9)$ & $6(6.8)$ & $3(8.8)$ & 0.815 \\
\hline \multicolumn{5}{|l|}{ Treatment at follow-up } \\
\hline$\beta$-blockers & $144(47.8)$ & $71(80.7)$ & $25(73.5)$ & $<0.0005$ \\
\hline Amiodarone & $25(8.3)$ & $12(13.7)$ & $6(17.6)$ & 0.045 \\
\hline Verapamil & $15(5)$ & $4(4.5)$ & $2(5.9)$ & 0.826 \\
\hline Diltiazem & $6(2)$ & $5(5.6)$ & $2(5.9)$ & 0.211 \\
\hline Disopyramide & $1(0.3)$ & $13(14.7)$ & $4(11.7)$ & $<0.0005$ \\
\hline Warfarin & $35(11.7)$ & $19(21.6)$ & $9(26.4)$ & 0.002 \\
\hline Aspirin/Clopidogrel & $52(17.3)$ & $20(22.7)$ & 7 (20.6) & 0.244 \\
\hline ICD implantation & $22(7.3)$ & $5(5.7)$ & $6(17.6)$ & 0.073 \\
\hline
\end{tabular}

Data given as $\mathrm{n}(\%)$ or mean $\pm \mathrm{SD}$. ${ }^{*} \mathrm{P}<0.05$ between HCM and LVOTO with Bonferroni analysis. ABPR, abnormal blood pressure response; $A F$, atrial fibrillation; ECG, electrocardiogram; $F H ~ H C M$, family history of hypertrophic cardiomyopathy; FH SCD, family history of sudden cardiac death; HCM, hypertrophic cardiomyopathy; ICD, implantable cardioverter defibrillator; LVOTO, left ventricular outflow tract obstruction; MVO, midventricular obstruction; MWT, maximum wall thickness; NSVT, non-sustained ventricular tachycardia; NYHA, New York Heart Association.

$0.3 \%$ of HCM patients and $0 \%$ of LVOTO patients). Coronary artery disease (CAD) was excluded as a cause of apical aneurysm formation according to the absence of significant coronary narrowing ( $>50 \%$ stenosis) in the left anterior descending artery on standard coronary angiography $(n=23)$; and no history resembling $\mathrm{CAD}$ and lack of any $\mathrm{CAD}$ risk factors for patients younger than 40 years old $(n=11)$. There were no significant differences between groups concerning factors predisposing to $\mathrm{CAD}$ such as hypertension $(\mathrm{P}=0.708)$ and diabetes mellitus ( $\mathrm{P}=0.688)$. There were also no significant differences concerning stroke prevalence and progression to end-stage HCM. Syncope ( 1 of the risk factors for sudden death) was significantly more frequent among MVO patients compared to HCM patients with and without LVOTO $(\mathrm{P}=0.03)$. Furthermore, the percentage of patients with $\geq 2$ risk factors for sudden death was greater in the MVO group (10/34, 29.4\%) compared to the LVOTO group $(16 / 88,18.1 \%)$ and HCM patients without obstruction $(26 / 301,8.6 \%$; $\mathrm{P}=0.005)$. As expected, treatment with negative inotropic agents, especially $\beta$-blockers and disopyramide, was mostly used in the obstruction groups (LVOTO and MVO; $\mathrm{P}<0.0005)$. During a median follow-up period of 84 months (range, 6-480 months), 5 of 34 patients with MVO (14.7\%) and 18 of 88 patients with LVOTO (20.5\%) underwent myectomy due to obstruction symptoms refractory to medical treatment, given that myectomy is more widely performed at AHEPA University Hospital compared to alcohol septal ablation. ${ }^{18}$ Among the operated MVO patients, 3 had concomitant MVO and LVOTO and underwent classical basal septal myectomy in order to relieve subaortic obstruction. The 2 patients with isolated MVO underwent extended mid-LV myectomy using a double approach technique (ie, trans-aortic and trans-apical). During the same period of time a total of 33 ICDs were implanted as primary or secondary prevention of sudden death, without significant differences in implantation rate between groups.

\section{Echocardiography}

Baseline echocardiographic characteristics of the HCM cohort are listed in Table 2. Patients with obstruction (either MVO or LVOTO) presented with smaller LV cavities both at sys- 


\begin{tabular}{|c|c|c|c|c|}
\hline & HCM $(n=301)$ & LVOTO (n=88) & MVO (n=34) & P-value \\
\hline LVEDD (cm) & $4.55 \pm 0.82$ & $4.15 \pm 0.77$ & $4.25 \pm 0.68$ & $<0.0005^{\star}$ \\
\hline LVESD $(\mathrm{cm})$ & $2.89 \pm 0.97$ & $2.21 \pm 0.65$ & $2.55 \pm 0.9$ & $0.001^{*}$ \\
\hline $\operatorname{LVEF}(\%)$ & $70 \pm 14$ & $76 \pm 10$ & $74 \pm 11.8$ & $0.001^{*}$ \\
\hline $\mathrm{LA}(\mathrm{cm})$ & $4.1 \pm 0.71$ & $4.6 \pm 0.7$ & $4.4 \pm 0.73$ & $<0.0005^{\star, \dagger}$ \\
\hline MWT (cm) & $1.97 \pm 0.5$ & $2.4 \pm 0.5$ & $2.2 \pm 0.7$ & $<0.0005^{\star, \dagger}$ \\
\hline MR (moderate to severe) & $14(4.7)$ & $23(26.1)$ & $9(26.5)$ & 0.017 \\
\hline \multicolumn{5}{|l|}{ Mitral Doppler } \\
\hline E wave $(\mathrm{m} / \mathrm{s})$ & $0.72 \pm 0.19$ & $0.88 \pm 0.33$ & $0.72 \pm 0.17$ & $<0.0005^{\star, \neq}$ \\
\hline A wave (m/s) & $0.71 \pm 0.27$ & $0.83 \pm 0.37$ & $0.7 \pm 0.27$ & $0.013^{*}$ \\
\hline$E / A$ & $1.12 \pm 0.5$ & $1.23 \pm 0.67$ & $1.24 \pm 0.68$ & 0.328 \\
\hline $\mathrm{E} / \mathrm{Em}$ & $12.4 \pm 5.5$ & $20.3 \pm 8.7$ & $15.5 \pm 4.3$ & $<0.0005^{\star, t, \neq}$ \\
\hline $\mathrm{E} / \mathrm{Ea}$ & $8.8 \pm 3.8$ & $14.5 \pm 7.4$ & $11.6 \pm 5.6$ & $<0.0005^{\star}, t, \neq$ \\
\hline \multicolumn{5}{|l|}{ Tric Doppler } \\
\hline $\mathrm{E} / \mathrm{A}$ & $1.15 \pm 0.37$ & $1.18 \pm 0.39$ & $1.1 \pm 0.56$ & 0.664 \\
\hline $\mathrm{E} / \mathrm{Er}$ & $4.4 \pm 1.8$ & $6.0 \pm 3.4$ & $4.8 \pm 2.3$ & $<0.0005^{\dagger}$ \\
\hline Mean resting gradients $(\mathrm{mmHg})$ & NA & $61.6 \pm 31.3$ & $65.6 \pm 31.1$ & NA \\
\hline Resting gradients after treatment $(\mathrm{mmHg})$ & NA & $32.5 \pm 30.4$ & $35 \pm 27.4$ & NA \\
\hline
\end{tabular}

Data given as $\mathrm{n}(\%)$ or mean $\pm \mathrm{SD}$. ${ }^{*} \mathrm{P}<0.05$ between HCM and LVOTO; ${ }^{\mathrm{t}} \mathrm{P}<0.05$ between HCM and MVO; $\mathrm{P}<<0.05$ between MVO and LVOTO after Bonferroni analysis. E/Ea, transmitral E wave to Ea wave obtained by tissue Doppler at the lateral corner of mitral annulus; $\mathrm{E} / \mathrm{Em}$, transmitral $\mathrm{E}$ wave to $\mathrm{Em}$ wave obtained by tissue Doppler at the septal corner of mitral annulus; E/Er, transtricuspid $\mathrm{E}$ wave to $\mathrm{Er}$ wave obtained by tissue Doppler at the lateral corner of tricuspid annulus; LA, left atrial diameter; LVEDD, left ventricular end-diastolic diameter; LVEF, left ventricular ejection fraction; LVESD, left ventricular end systolic diameter; MR, mitral regurgitation. Other abbreviations as in Table 1.

Table 3. Cox Regression Analysis for Surrogate Sudden Death and Combined Cardiovascular Endpoints in $\mathrm{HCM}$

Status No. patients

Sudden death surrogate endpoint

ABPR

Yes

No

57

366

Syncope

Yes

No

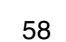

No. events

Univariate Cox analysis

(\%)

Crude HR
$(95 \% \mathrm{Cl})$

Multivariate Cox analysis

MWT >3.0

Yes 30

No

393

FH SCD

Yes

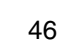

No

377

NSVT

Yes 43

No $\quad 380$

MVO

Yes $\quad 34$

$4(7)$

$1.26(2.35-3.7)$

0.678

$19(5.2)$

11 (19)

$12(3.3)$

$5.46(2.4-12.5)$

$<0.0005$

$3.2(1.33-7.6)$

0.009

$6(20)$

$3.36(1.3-8.6)$

0.012

$2.4(0.9-6.3)$

0.079

$17(4.3)$

$$
8(17.4)
$$

$3.7(1.55-8.8)$

0.003

$2.8(1.1-6.9)$

0.029

$15(4)$

8 (18.6)

$4.42(1.86-10.5)$

0.001

$2.7(1.2-6.9)$

0.033

15 (3.9)

$$
6 \text { (17.6) }
$$

$4.3(1.69-10.9)$

0.004

$3.3(1.26-8.85)$

0.016

Combined cardiovascular endpoint

Age at diagnosis

NA

NYHA class

NA
MVO
Yes
No

NA

NA

34

389

17 (4.4) (95\% Cl)

P-value 
A

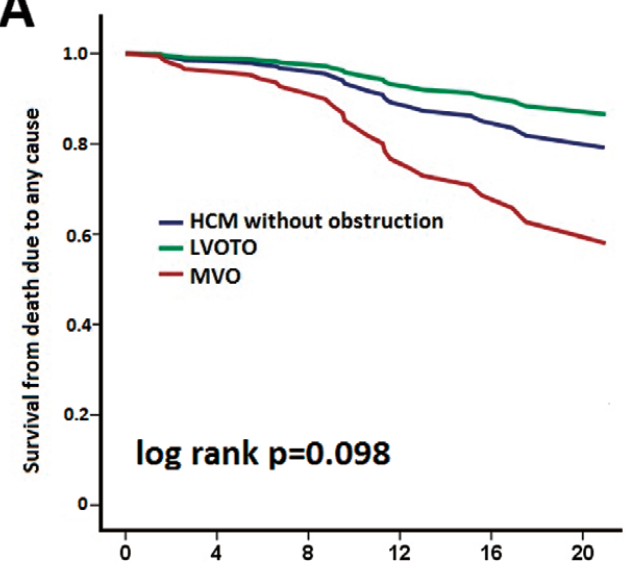

Number at risk HCM $301 \quad 252$ \begin{tabular}{lll} 
LVOTO & 88 & 79 \\
\hline
\end{tabular}

C

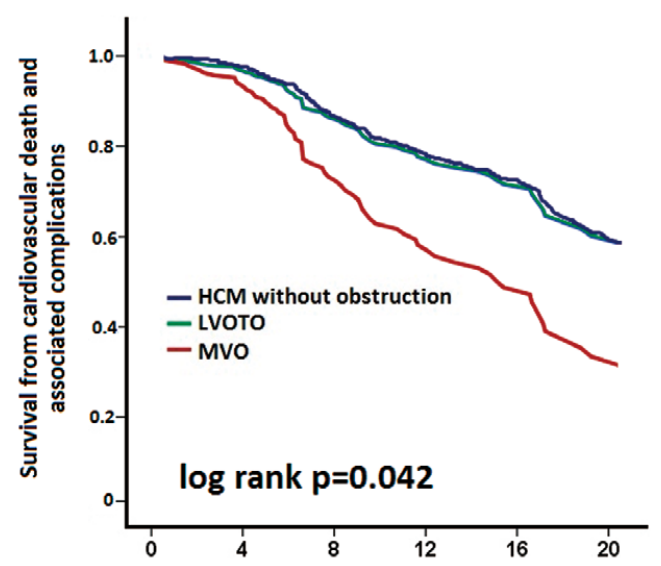

Number at risk

$\begin{array}{lcccccc}\text { HCM } & 301 & 252 & 93 & 69 & 46 & 23 \\ \text { LVOTO } & 88 & 79 & 41 & 22 & 16 & 10 \\ \text { MVO } & 34 & 29 & 15 & 8 & 4 & 2\end{array}$

B

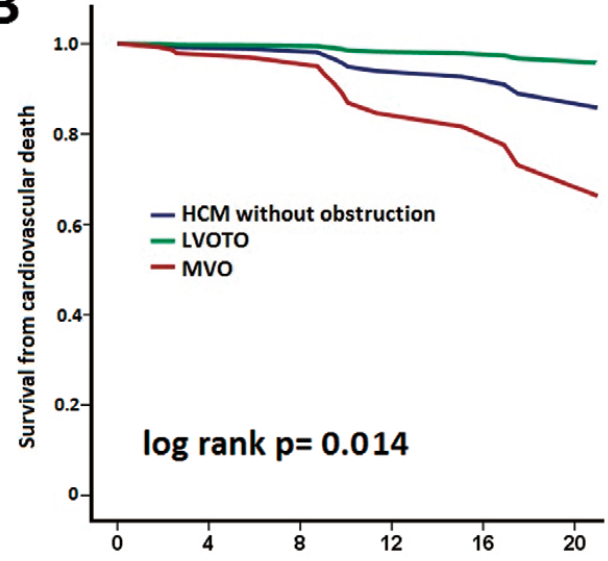

Follow up in years

$\begin{array}{lcccccc}\text { HCM } & 301 & 252 & 93 & 69 & 46 & 23 \\ \text { LVOTO } & 88 & 79 & 41 & 22 & 16 & 10 \\ \text { MVO } & 34 & 29 & 15 & 8 & 4 & 2\end{array}$

D

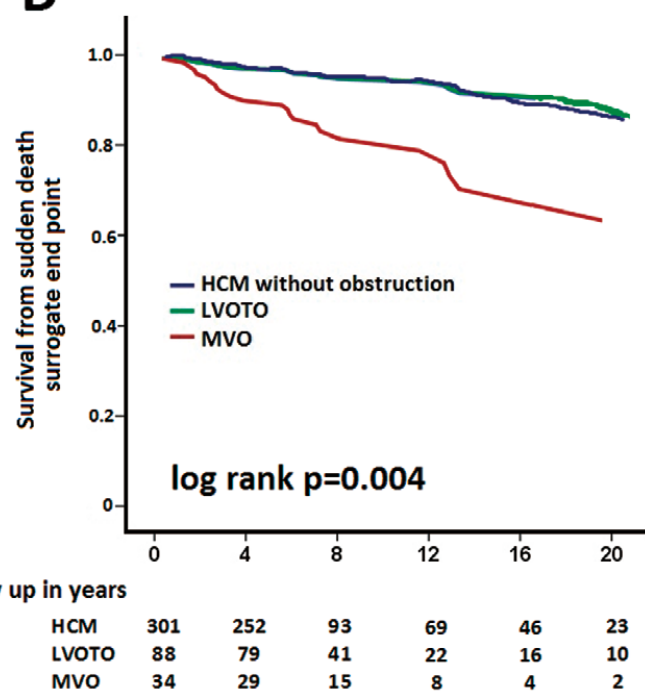

Figure 3. Kaplan-Meier curves showing the proportions of patients with hypertrophic cardiomyopathy (HCM), left ventricular outflow tract obstruction (LVOTO) and midventricular obstruction (MVO) experiencing adverse events or reaching study endpoints during follow-up. (A) Survival from overall mortality; (B) rate of survival from cardiovascular death (including death due to heart failure, stroke or sudden death). (C) Event-free rates for combined cardiovascular endpoint fulfilled by patients who experienced progression to end-stage HCM and died due to heart failure. (D) Survival from the surrogate sudden death endpoint, which consisted of sudden death events along with potentially lethal arrhythmic incidents such as ventricular tachycardia or fibrillation, resuscitated cardiac arrest and appropriate implantable defibrillator discharge. $\mathrm{P}<0.05$ was considered statistically significant. Interpolation lines were used to smooth survival curves.

tole and diastole compared to HCM patients without obstruction $(\mathrm{P}<0.005)$. The increased intracavitary pressures due to obstruction resulted in larger left atrial dimensions in both obstruction groups, while both MVO and LVOTO groups had more advanced stages of diastolic dysfunction (restrictive physiology patterns were recognized in: $6 / 34,17.6 \%$ in the MVO group; $11 / 88,12.5 \%$ in the LVOTO group; and 21/301, $7 \%$ in the HCM patients without obstruction; $\mathrm{P}=0.03$ ), even though patients with LVOTO tended to develop earlier more elevated filling pressures, demonstrating higher E/Em and E/ Ea ratios.

\section{Outcomes}

During follow-up 29 of the present patients $(6.9 \%)$ died due to any reason. In the MVO group 6 out of 34 patients died due to cardiovascular death (17.6\%), among them 3 died suddenly $(8.8 \%)$. Regarding the surrogate sudden death endpoint, 6 patients with MVO (17.6\%) experienced sudden death-relevant events, including 3 patients with sudden death, 2 patients with appropriate defibrillator discharges and 1 patient with successfully resuscitated cardiac arrest due to ventricular fibrillation. Five-year survival from all-cause mortality reached $93.8 \%$ (95\% CI: 89.5-98.1) for the MVO group and did not differ 


\begin{tabular}{|c|c|c|c|}
\hline & $\begin{array}{c}\text { MVO (+) } \\
\text { aneurysm }(n=9)\end{array}$ & $\begin{array}{c}\text { MVO (-) } \\
\text { aneurysm }(n=25)\end{array}$ & P-value \\
\hline Gender (male) & $2(22.2)$ & $14(56)$ & 0.125 \\
\hline Age at diagnosis (years) & $44.6 \pm 15$ & $52.1 \pm 17$ & 0.336 \\
\hline Age at first evaluation (years) & $50.3 \pm 13.2$ & $56.7 \pm 17.6$ & 0.25 \\
\hline Symptoms at presentation & $1(11.1)$ & $0(0)$ & 0.265 \\
\hline Normal ECG & $0(0)$ & $1(4)$ & 1.0 \\
\hline $\mathrm{FH} \mathrm{HCM}$ & $6(66.7)$ & $5(20)$ & 0.033 \\
\hline \multicolumn{4}{|l|}{ SCD risk factors } \\
\hline MWT >3.0 (cm) & $1(11.1)$ & $3(12)$ & 1.0 \\
\hline NSVT & $2(22.2)$ & $5(20)$ & 1.0 \\
\hline ABPR & $2(22.2)$ & $4(16)$ & 0.644 \\
\hline Syncope & $2(22.2)$ & $7(28)$ & 1.0 \\
\hline FH SCD & $4(44.4)$ & $3(12)$ & 0.061 \\
\hline \multicolumn{4}{|l|}{ NYHA class diagnosis } \\
\hline 1 & $1(11.1)$ & $2(8)$ & 0.855 \\
\hline II & $6(66.7)$ & $14(56)$ & \\
\hline III & $2(22.2)$ & $8(32)$ & \\
\hline IV & $0(0)$ & $1(4)$ & \\
\hline \multicolumn{4}{|l|}{$\mathrm{AF}$} \\
\hline Paroxysmal & $5(55.6)$ & $7(28)$ & 0.224 \\
\hline Permanent & $0(0)$ & $2(8)$ & 1.0 \\
\hline Progression to end-stage $\mathrm{HCM}$ & $4(44.4)$ & $0(0)$ & 0.003 \\
\hline Stroke & $0(0)$ & $3(12)$ & 0.549 \\
\hline \multicolumn{4}{|l|}{ Treatment at follow-up } \\
\hline$\beta$-blockers & $7(77.7)$ & $18(72)$ & 0.853 \\
\hline Amiodarone & $2(22.2)$ & $4(16)$ & 0.65 \\
\hline Verapamil & $1(11)$ & $1(4)$ & 0.465 \\
\hline Diltiazem & $0(0)$ & $2(8)$ & 0.682 \\
\hline Disopyramide & $0(0)$ & $4(16)$ & 0.442 \\
\hline Warfarin & $3(33.3)$ & $6(24)$ & 0.862 \\
\hline Aspirin/Clopidogrel & $3(33.3)$ & $4(16)$ & 0.253 \\
\hline ICD implantation & 3 (33.3) & $3(12)$ & 0.306 \\
\hline
\end{tabular}

Data given as $\mathrm{n}(\%)$ or mean $\pm \mathrm{SD}$. Abbreviations as in Table 1 .

significantly compared to $98.8 \%$ (95\% CI: 97.6-99.9) for the LVOTO group and $98.5 \%$ (95\% CI: 97.8-99.1) for the HCM patients without obstruction (log-rank $\mathrm{P}=0.098$ ). Accordingly, 5-year survival from cardiovascular death was 93.5\% (95\% CI: 89.1-97.9) for the MVO group and it was lower compared to 96.2\% (95\% CI: 92.4-99.9) for the LVOTO group and $98.9 \%$ (95\% CI: 98.2-99.4) for the non-obstruction group (log-rank $\mathrm{P}=0.014)$. The probability of the surrogate cardiovascular endpoint among patients with MVO was significantly higher (5year survival, 96.9\%; 95\% CI: 93.8-99) compared to patients without obstruction (5-year survival, 99.3\%; 95\% CI: 98.8 99.8) and patients with LVOTO (5-year survival, 98.6\%; $95 \%$ CI: 97.2-99.9; $\log$-rank $\mathrm{P}=0.042$ ). Finally, on univariate analysis, patients with MVO had a significantly greater likelihood of reaching sudden death surrogate endpoint compared to the other 2 groups of patients (log-rank $\mathrm{P}=0.004$; Figure 3 ). Univariate and multivariate Cox regression analysis results are given in Table 3. By entering the established 5 risk factors for sudden death and MVO in a multivariate Cox regression model (except for abnormal blood pressure response, which tended to be a non-significant predictor on univariate analysis) for predicting sudden death surrogate endpoint, MVO was identified as an independent determinant of this combined endpoint (adjusted HR, 3.3; 95\% CI: 1.26-8.85, $\mathrm{P}=0.016$ ). Similarly, by entering age at diagnosis, NYHA class during first evaluation and MVO in another multivariate Cox regression model for predicting cardiovascular surrogate endpoint, patients with MVO were found to have a 2.62-fold greater risk for presenting with progression to end-stage HF or HF-related death compared to non-MVO groups (adjusted HR, 2.62; 95\% CI: 1.2-8.8, $\mathrm{P}=0.047)$.

\section{MVO and LVOTO}

The patients with both MVO and LVOTO did not differ compared to MVO patients concerning gender $(\mathrm{P}=0.163)$, symptom status and NYHA class at presentation $(\mathrm{P}=1.0$ and $\mathrm{P}=0.233$, respectively) as well as progression to end-stage $\mathrm{HCM}(\mathrm{P}=0.126)$. They also did not differ significantly concerning other parameters such as age at diagnosis, frequency of established sudden death risk factors, stroke incidence and treatment application during follow-up. The major difference between the 2 groups concerned the formation of apical aneurysms, because all 9 aneurysms recorded presented in patients with MVO $(9 / 20$, $45 \%)$ and not coexisting LVOTO $(\mathrm{P}=0.004)$.

\section{MVO and Apical Aneurysm}

All 9 patients with apical aneurysms and MVO were identified on cardiac MRI and echocardiography. Table 4 lists the base- 
line demographic and clinical characteristics of MVO patients with presence $(n=9)$ or absence $(n=25)$ of apical aneurysms and the comparison between groups. MVO patients with an aneurysm had a higher probability to progress to end-stage $\mathrm{HCM}(\mathrm{P}=0.003)$. During follow-up, 4 of 9 patients with apical aneurysm and MVO (44.4\%) and 2 of 25 patients without aneurysm (8\%) experienced progression to end-stage HCM (burnt out) or death due to $\mathrm{HF}$, with the survival rates from $\mathrm{HF}$ and associated death being significantly lower in the aneurysm group (5-year survival: $88.9 \%, 95 \% \mathrm{CI}$ : 78.4-99.4 for the MVO group without aneurysm; 74.1\%, 95\% CI: 58-90.2 for the aneurysm group; log-rank $\mathrm{P}=0.032$ ). In contrast, non-significant differences were recorded for survival from sudden death and surrogate endpoints (5-year survival: $87 \%, 95 \% \mathrm{CI}$ : 80-94 for the non-aneurysm group; 77.8\%, 95\% CI: 64.4-91.6 for the MVO group with apical aneurysm; $\log$-rank $\mathrm{P}=0.724)$, and for survival from cardiovascular death $(\mathrm{P}=0.748)$.

\section{Discussion}

A total of $8 \%$ of patients with HCM in the present single-center cohort had MVO. This is similar to that noted in previous studies, which have reported the occurrence of MVO to be between $9 \%$ and $12.9 \%$ in HCM cohorts. ${ }^{10,19-21}$ The minor differences reported could be due to racial or ethnic variations, selection bias (an unavoidable limitation of all large-scale HCM trials), different inclusion criteria or definitions used for MVO, and possibly due to the fact that some older studies (which have reported an even lower incidence of MVO of $1 \%)^{9}$ have mainly described apical aneurysm formation frequency rather than MVO existence itself. It is evident that routine use of MRI and focused echocardiographic techniques, along with a high level of suspicion, have facilitated recognition and diagnosis of MVO in HCM patients.

Patients with MVO have been far more symptomatic at first presentation, compared to HCM patients without obstruction or with LVOTO, with dyspnea being the dominating symptom. Additionally, a left ventricular apical aneurysm was identified in approximately one-fourth of HCM patients with MVO, a result confirmed by previous studies, also suggesting the existence of a close overlap between MVO and apical aneurysm in patients with HCM.9,11 The exact mechanism leading to formation of apical aneurysms in patients with MVO remains currently undetermined, but it is hypothesized that increased intracavitary gradient imposes an increased pressure load on the apical myocardium, increasing metabolic demand and reducing oxygen supply, mainly by extravascular compression of the coronary arteries. Increased diastolic pressures in the isolated left ventricular apex, generating also diastolic paradoxic jet flow identified on echocardiography, have been noted in a previous study. ${ }^{21}$ Thus, this indirect coronary flow reserve decline results in chronic myocardial ischemia, diffuse fibrosis in the affected apical region and finally aneurysm formation. ${ }^{9}$ Identification of identical apical aneurysms in the same family of patients ( 2 sisters and daughter/nephew) supports the potential role of genetic substrate/predisposition in formation of aneurysms. ${ }^{10}$ The present study did not record any significant differences in mid-cavitary gradients between patients with MVO and/or without an apical aneurysm. Taking into consideration the systolic impairment of apex after aneurysm formation, it is reasonable to assume that midventricular gradients must have been higher in the aneurysm group, gradually fading to levels equal to those recorded in patients with MVO but without apical aneurysms. It would be interesting to trace the effect of negative inotropic agents and or myectomy results in mid-cavitary gradients and consequently in apical aneurysm formation in patients with MVO diagnosed early enough to benefit from therapeutic intervention.

Progression to end-stage HCM along with mortality from HF has been recognized more frequently among patients with MVO and apical aneurysms compared to HCM patients with or without LVOTO. Even compared to MVO patients without apical aneurysm, patients presenting with apical aneurysm formation had a more unfavorable course in terms of HF progression and HF-related death. Transmural apical myocardial scarring and concomitant aneurysm formation lead to primary regional systolic impairment, alteration of local and secondarily global hemodynamic conditions, cavity dilatation as a compulsory remodeling mechanism similar to that observed after myocardial infarction, ${ }^{22}$ systolic dysfunction and finally development of a dilated phenotype. This pathophysiologic cascade is further supported by the fact that a decline or even disappearance of MVO gradient in MVO patients progressing towards a dilated phenotype has been noted in the present cohort. Even though no single pathophysiologic mechanism can explain HF progression in HCM patients, it is likely that MVO patients with apical aneurysm formation develop mostly systolic impairment and consequently systolic HF; at the same time, patchy or disperse fibrosis observed in patients with LVOTO ${ }^{22}$ leads to diastolic dysfunction, impaired relaxation patterns, elevated end-diastolic pressure and finally settlement of diastolic HF. Echo indices (such as E/Ea) recorded in the present patients support previous hypothesis. Additionally, previous studies strongly support the present results, ${ }^{9}$ because patients with MVO and apical aneurysms have demonstrated a largely unfavorable clinical course, experiencing progression to end-stage HF and HF-related death.

A key point in the present analysis, however, remains the relatively high incidence of sudden death and associated arrhythmic events among patients with MVO, irrespective of apical aneurysm formation. Six of 34 patients with MVO reached, during long-term follow-up, the surrogate sudden death endpoint, while MVO turned out to be a significant independent predictor of arrhythmic events/deaths, far beyond the existence of the 5 established clinical risk factors for sudden death. Compared to the Minami et al study, which mainly associated arrhythmic events with the presence of apical aneurysms, ${ }^{12}$ the present study suggests that hypertrophied and, in varying degree, fibrotic midventricular segments (as shown by LGE on MRI in the present patients) serve as a potential arrhythmogenic substrate facilitating re-entry phenomena, earlier than or independently of aneurysm formation. ${ }^{23,24}$ In that case, MVO could be considered as a risk factor for sudden death, and therapeutic interventions in this group should possibly be modified to include primary prevention of sudden death and arrhythmic adverse events, with the use of an implanted defibrillator. ${ }^{25}$ Additional clinical studies are needed to clarify if MVO alone, or appropriate defibrillator discharge rate in patients presenting with MVO as a sole risk factor for sudden death, justify the prophylactic use of ICD.

\section{Study Limitations}

Retrospective enrollment of HCM patients coming from a single tertiary center could be regarded as an unavoidable limitation affecting all large-scale clinical HCM studies. The present study could not estimate the effect of negative inotropic agents used at study endpoints. In Europe during the last decade, use of disopyramide is the next-step medication for patients with severe obstructive symptoms not well tolerated or undertreated with $\beta$-blocker or calcium antagonist. ${ }^{26}$ De- 
spite that, disopyramide use was limited in the present cohort because the majority of patients with obstruction responded well to $\beta$-blockers and calcium antagonists (as monotherapies or combination thereof), with patients suffering from more severe symptoms finally preferring invasive treatment to disopyramide, due to more frequent side-effects or increased proarrhythmia risk connected with its use. ${ }^{26,27}$ Furthermore, the number of patients with MVO having undergone myectomy was low and their after-surgery follow-up period was too short to estimate potential benefits on survival or endpoint frequency rates. Finally, MRI was not performed in all patients in the MVO group. Performance of MRI in all MVO patients would probably identify more apical aneurysms, while more LGE data could support even more the hypotheses on progression to burnt out HCM and sudden death in MVO patients.

\section{Conclusions}

MVO was recognized in approximately 1 in every 12 patients in the present HCM cohort and was identified as a strong, independent predictor of sudden death and associated lethal arrhythmic events as well as a determinant of progression to end-stage HCM and HF-related death. Apical aneurysms were discovered in approximately one-fourth of patients with MVO, being an almost exclusive characteristic of the group. The present study has shown that patients with MVO and apical aneurysm formation present with an even worse course concerning progression to burnt out HCM and HF-related death compared to patients with MVO without aneurysm. In contrast, there were no significant differences in survival from sudden death and arrhythmic events between patients with and without aneurysm in the MVO group. Further studies are required to record the potential benefits of myectomy on these patients and further therapeutic regimens that could be used.

\section{References}

1. Otsuka H, Arimura T, Abe T, Kawai H, Aizawa Y, Kubo T, et al. Prevalence and distribution of sarcomeric gene mutations in Japanese patients with familial hypertrophic cardiomyopathy. Circ J 2012; 76: 453-461.

2. Kubo T, Kitaoka H, Okawa M, Baba Y, Hirota T, Hayato K, et al. Genetic screening and double mutation in Japanese patients with hypertrophic cardiomyopathy. Circ J 2011; 75: 2654-2659.

3. Gersh BJ, Maron BJ, Bonow RO, Dearani JA, Fifer MA, Link MS, et al. 2011 ACCF/AHA Guideline for the Diagnosis and Treatment of Hypertrophic Cardiomyopathy: A report of the American College of Cardiology Foundation/American Heart Association Task Force on Practice Guidelines. Developed in collaboration with the American Association for Thoracic Surgery, American Society of Echocardiography, American Society of Nuclear Cardiology, Heart Failure Society of America, Heart Rhythm Society, Society for Cardiovascular Angiography and Interventions, and Society of Thoracic Surgeons. J Am Coll Cardiol 2011; 58: e212-e260, doi:10.1016/j.jacc.2011.06.011 (accessed December 14, 2012).

4. Falicov RE, Resnekov L, Bharati S, Lev M. Mid-ventricular obstruction: A variant of obstructive cardiomyopathy. Am J Cardiol 1976; 37: $432-437$

5. Falicov RE, Resnekov L. Midventricular obstruction in hypertrophic obstructive cardiomyopathy: New diagnostic and therapeutic challenge. Br Heart J 1977; 39: 701 -705.

6. Blazer D, Kotler MN, Parry WR, Wertheimer J, Nakhjavan FK. Noninvasive evaluation of mid-left ventricular obstruction by twodimensional and Doppler echocardiography and color flow Doppler echocardiography. Am Heart J 1987; 114: 1162-1168.

7. Tse HF, Ho HH. Sudden cardiac death caused by hypertrophic cardiomyopathy associated with midventricular obstruction and apical aneurysm. Heart 2003; 89: 178.

8. Efthimiadis GK, Giannakoulas G, Parcharidou DG, Ziakas AG, Papadopoulos CE, Karoulas T, et al. Subaortic and midventricular obstructive hypertrophic cardiomyopathy with extreme segmental hypertrophy. Cardiovasc Ultrasound 2007; 5: 12.

9. Maron MS, Finley JJ, Bos JM, Hauser TH, Manning WJ, Haas TS, et al. Prevalence, clinical significance, and natural history of left ventricular apical aneurysms in hypertrophic cardiomyopathy. Circulation 2008; 118: $1541-1549$.

10. Efthimiadis GK, Pliakos C, Pagourelias ED, Parcharidou DG, Spanos G, Paraskevaidis S, et al. Hypertrophic cardiomyopathy with midventricular obstruction and apical aneurysm formation in a single family: Case report. Cardiovasc Ultrasound 2009; 7: 26.

11. Shah A, Duncan K, Winson G, Chaudhry FA, Sherrid MV. Severe symptoms in mid and apical hypertrophic cardiomyopathy. Echocardiography 2009; 26: 922-933.

12. Minami Y, Kajimoto K, Terajima Y, Yashiro B, Okayama D, Haruki $\mathrm{S}$, et al. Clinical implications of midventricular obstruction in patients with hypertrophic cardiomyopathy. J Am Coll Cardiol 2011; 57: 2346-2355.

13. Efthimiadis GK, Parcharidou D, Pagourelias ED, Meditskou S, Spanos G, Hadjimiltiades S, et al. Prevalence and clinical outcomes of incidentally diagnosed hypertrophic cardiomyopathy. Am J Cardiol 2010; 105: $1445-1450$.

14. Maron BJ, Gottdiener JS, Epstein SE. Patterns and significance of distribution of left ventricular hypertrophy in hypertrophic cardiomyopathy. A wide angle, two dimensional echocardiographic study of 125 patients. Am J Cardiol 1981; 48: 418-428.

15. Klues HG, Schiffers A, Maron BJ. Phenotypic spectrum and patterns of left ventricular hypertrophy in hypertrophic cardiomyopathy: Morphologic observations and significance as assessed by two-dimensional echocardiography in 600 patients. J Am Coll Cardiol 1995; 26: $1699-1708$.

16. Panza JA, Petrone RK, Fananapazir L, Maron BJ. Utility of continuous wave Doppler echocardiography in the noninvasive assessment of left ventricular outflow tract pressure gradient in patients with hypertrophic cardiomyopathy. J Am Coll Cardiol 1992; 19: 91-99.

17. Spiewak M, Malek LA, Misko J, Chojnowska L, Milosz B, Klopotowski $\mathrm{M}$, et al. Comparison of different quantification methods of late gadolinium enhancement in patients with hypertrophic cardiomyopathy. Eur J Radiol 2010; 74: e149-e153, doi:10.1016/j.ejrad.2009.05.035 (accessed December 14, 2012).

18. Rigopoulos AG, Seggewiss H. A decade of percutaneous septal ablation in hypertrophic cardiomyopathy. Circ J 2011; 75: 28-37.

19. Fighali S, Krajcer Z, Edelman S, Leachman RD. Progression of hypertrophic cardiomyopathy into a hypokinetic left ventricle: Higher incidence in patients with midventricular obstruction. J Am Coll Cardiol 1987; 9: 288-294.

20. De Gregorio C. Left ventricular dynamic obstruction by atypical papillary muscle morphology: Is this finding so unusual in clinical practise? J Am Soc Echocardiogr 2007; 20: 100-101.

21. Nakamura T, Matsubara K, Furukawa K, Azuma A, Sugihara H, Katsume $\mathrm{H}$, et al. Diastolic paradoxic jet flow in patients with hypertrophic cardiomyopathy: Evidence of concealed apical asynergy with cavity obliteration. J Am Coll Cardiol 1992; 19: 516-524.

22. Opie LH, Commerford PJ, Gersh BJ, Pfeffer MA. Controversies in ventricular remodelling. Lancet 2006; 367: 356-367.

23. Adabag AS, Maron BJ, Appelbaum E, Harrigan CJ, Buros JL, Gibson $\mathrm{CM}$, et al. Occurrence and frequency of arrhythmias in hypertrophic cardiomyopathy in relation to delayed enhancement on cardiovascular magnetic resonance. J Am Coll Cardiol 2008; 51: 1369-1374.

24. Gerstenfeld EP. Hypertrophic cardiomyopathy with midventricular obstruction: Another substrate for ventricular tachycardia? J Cardiovasc Electrophysiol 2010; 21: 1000-1001.

25. Maron BJ. Risk stratification and role of implantable defibrillators for prevention of sudden death in patients with hypertrophic cardiomyopathy. Circ J 2010; 74: 2271-2282.

26. Sherrid MV, Arabadjian M. A primer of disopyramide treatment of obstructive hypertrophic cardiomyopathy. Prog Cardiovasc Dis 2012; 54: 483-492.

27. Sherrid MV, Barac I, McKenna WJ, Elliott PM, Dickie S, Chojnowska $\mathrm{L}$, et al. Multicenter study of the efficacy and safety of disopyramide in obstructive hypertrophic cardiomyopathy. J Am Coll Cardiol 2005; 45: $1251-1258$. 